\title{
The effect of obsessive compulsive symptoms on psychopathology in patients with schizophrenia
}

\author{
Ng Boon Seng 1 , Anne Yee 1 , Mahmoud Danaee², Loh Huai Seng ${ }^{3}$, Stephan Thevananthan Jambunathan 1
}

${ }^{1}$ Department of Psychological Medicine, Department of Psychological Medicine, University Malaya, Kuala Lumpur, Malaysia.

${ }^{2}$ Academic Development Centre (ADEC), University Malaya, Kuala Lumpur, Malaysia.

${ }^{3}$ Clinical Academic Unit, Newcastle University Medicine Malaysia, Nusajaya, Johor, Malaysia.

Received: 06/21/2017 - Accepted: 06/06/2018

DOI: 10.1590/0101-60830000000159

\begin{abstract}
Background: There is a growing interest on the impact of comorbid obsessive-compulsive symptoms (OCS) on the course and severity of schizophrenia in recent years. Objectives: This study determined the prevalence of OCS in schizophrenia patients and the clinical outcomes of the comorbidity. Methods: A total of 220 schizophrenia patients were recruited. All the participants completed Structure Clinical Interview version, Yale Brown Obsessive Compulsive Scale, Calgary Depression Scale for Schizophrenia, Columbia Suicide Severity Rating Scale and World Health Organization Quality of Life - Brief Version (WHOQOL-BREF). Results: Significantly higher number of schizophrenia patients with OCS were taking Clozapine $(\mathrm{p}=0.023)$ and antidepressants $(\mathrm{p}=0.013)$. Schizophrenia patients with OCS showed more severe positive $(\mathrm{p}<0.001)$ and general symptoms $(\mathrm{p}<0.001)$ of schizophrenia, higher depressive symptoms $(\mathrm{p}=0.013)$, higher suicidality $(\mathrm{p}<0.001)$, more hospitalization $(\mathrm{p}=0.044)$, poorer physical $(\mathrm{p}=0.034)$ and psychological $(\mathrm{p}=0.032)$ domain in WHOQOLBREF. Discussion: Schizophrenia patients with OCS are associated with more severe psychopathology and depressive symptoms which subsequently suffered poorer physical and psychological health. Hence, recognition of OCS in schizophrenia and early initiation of effective treatment may be able to reduce the burden for people with chronic mental illness.
\end{abstract}

Seng NB et al. / Arch Clin Psychiatry. 2018;45(3):61-6

Keywords: Schizophrenia, obsessive compulsive symptoms, depression, quality of life.

\section{Introduction}

Schizophrenia is a long-term serious psychotic disorder characterized by main clinical features of hallucinations, delusions, disorganized thoughts, changes in behavior, and negative symptoms. Over the years, clinicians and researchers paid less attention to nonpsychopathological manifestations among the schizophrenia patients, one of which is the obsessive-compulsive symptoms (OCS). Despite a wide variation of prevalence noted in many parts of the world, ranging from $1.1 \%$ to $50 \%^{1}$, a more recent study observed that the pooled prevalence was $30.7 \%{ }^{2}$. OCS in schizophrenia were once thought to be rare and benign in nature, however some recent studies have shown not only greater prevalence rate but poorer outcome among these patients ${ }^{3}$. Interestingly, many researchers were facing difficulties to generalize the findings as there were no standard criteria available to determine the presence of clinically significant OCS, as compared to obsessive compulsive disorder which can be clearly categorized in Diagnostic and Statistical Manual of Mental Disorder (DSM).

In the past, obsession had not only been postulated to protect against psychosis and thought disorganization ${ }^{4}$ but also believed to prevent "personality disintegration" in schizophrenia". However, these findings were not reproducible in subsequent studies, which found that schizophrenia with OCS had actually worse outcomes ${ }^{6,7}$. Two years ago, a group of researchers again proved that patients suffering from schizophrenia with OCS had significantly higher scores in both the Positive and Negative Syndrome Scale (PANSS) and Beck Depression Inventory (BDI) when compared to the nonOCS groups. Furthermore, higher rates of suicidal plans or attempts were also found among these patients ${ }^{9,10}$. There were findings from various studies to suggest that schizophrenia with OCS might be a distinct subtypes of schizophrenia ${ }^{7,11,12}$; and that OCS itself was considered as one of the core clinical features and symptom domains of schizophrenia rather than being an additional clinical condition'.
There are growing evidence to suggest the existence of a schizo-obsessive compulsive disorder (Schizo-OCD) subtype of schizophrenia. This subtype is shown to manifest different neuropsychological and clinical outcomes among the schizophrenia patients. A proposed diagnostic criteria for Schizo-OCD was available since $2012^{13}$. In that proposed criteria, criterion A of OCD must be present at some point in time during the course of schizophrenia. In addition, the obsession/compulsion must be present in substantial amount of time and must not be related to the delusion or hallucination from schizophrenia. Neurological soft signs (NSS) were defined as "minor neurological signs which reflect dysfunction in areas of motor coordination, integrative sensory function and ordering complex motor tasks, but the dysfunctions are not localizable to specific brain structure"14,15. Studies showed that Schizo-OCD scored higher in the Neurological Evaluation Scale (NES) compared to healthy controls ${ }^{16,17}$, but no difference when compared with schizophrenia alone. These findings have further suggested that Schizo-OCD may be a distinct subtype of schizophrenia, and not merely a more severe form of OCD. In addition, previous studies had consistently reported there were no significant differences between OCS in obsessive compulsive disorder (OCD) and Schizo-OCD in term of clinical characteristics ${ }^{18,19}$. Faragian et al. reported that the symptoms in Schizo-OCD were comparable to those revealed in "pure" OCD. The author concluded that the universal mechanisms were involved in the pathogenesis of OCD regardless of the presence of schizophrenia ${ }^{18,19}$.

Contradictions on the effects of atypical anti-psychotic (AAPs) medications such as clozapine on schizophrenic patients still exist. The risk of AAPs-induced OCS has been reported in many studies $20-22$. AAPs with higher serotonergic activities has higher propensities in inducing $\mathrm{OCS}^{23}$. Clozapine stands out as the most frequently reported AAPs to induce OCS due to its highest propensity in serotonergic activities ${ }^{24,25}$. In contrary, existing literatures also showed 
that AAPs such as Aripiprazole and Risperidone to be effective in the augmentation of selective serotonin reuptake-inhibitor (SSRI)resistant $\mathrm{OCD}^{26,27}$. In addition, AAPs with strong dopamine receptor 2 (D2) blockade were reported to be effective in treating Schizo-OCD.

Some studies, however, found no significant difference between schizophrenia with or without OCS in terms of their psychopathology and suicidality $28-30$. We believe that this finding could have been a result of different methodological approach. Studies which recruited inpatients tended to reflect a higher severity in psychopathological domains $s^{6,7}$ as compared to those which studied on patients recruited from the outpatient settings ${ }^{28,29}$. During acute episodes of psychosis, it would also be very difficult to discern obsessions from delusions of schizophrenia ${ }^{31}$. In addition, some studies had used different definitions for clinically significant OCS resulted in a non-homogenous recruitment of study participants $\mathrm{s}^{8,32}$, while some researchers had shown that both obsessive-compulsive disorder (OCD) and schizophrenia with OCS were no different in terms of their obsessive and compulsive symptom structures ${ }^{18}$.

Since the co-occurrence of OCS and schizophrenia had always been an interesting topic of discussion and a real challenge faced by the treating clinicians, but studies had mainly been done in the western countries, we decided to conduct this study in Malaysia to determine the prevalence of OCS among a group of stable schizophrenia patients attending a tertiary hospital outpatient clinic. In addition, our study also intended to resolve any conflicting evidence in literatures and examine the clinical variables of OCS in schizophrenia.

\section{Methods}

\section{Subjects}

In this cross-sectional study, participants consisted of stable outpatients who were attending their follow up sessions in the psychiatric clinic in University Malaya Medical Center (UMMC) - a tertiary hospital situated in Kuala Lumpur, Malaysia - between August 2014 and July 2015. All recruited participants were diagnosed with schizophrenia based on Diagnostic and Statistical Manual for Mental Disorder, Fifth Edition (DSM-V), understood English or Malay language and were on the same antipsychotic treatment for at least six months. Patients were excluded if (1) they were having active psychotic symptoms, which would affect their capacity for informed consent; (2) they had intellectual disability and dementia, and (3) patients who refused to participate. The study was approved by the UMMC medical ethics committee. All participants were thoroughly briefed on the study protocol and their informed consent obtained. After participants' demographic data were obtained from the case files, all the participants were assessed by the author using Yale Brown Obsessive Compulsive Scale (YBOCS) Symptoms Checklist, YaleBrown Obsessive Compulsive Scale (YBOCS), Positive and Negative Symptoms for Schizophrenia (PANSS), Calgary Depression Rating Scale (CDSS), Columbia Suicide Severity Rating Scale (C-SSRS) and World Health Organization Quality of Life Scale Brief Malay Version (WHOQOL-BREF).

\section{Demographic variables}

The socio-demographic questionnaire was used to record relevant information about the participants of this study which included age, gender, ethnic group, marital status, education level, religion, employment status, family mental history, duration of illness and type of medication. Participants were instructed to complete the questionnaire by filling in the blanks and selecting one response that best described them.

\section{Obsessive compulsive symptomatology}

Obsessive compulsive symptoms (OCS) were first screened with Yale Brown Obsessive Compulsive Scale (YBOCS) Symptoms Checklist. Subsequently, patients were assessed with YBOCS $^{33}$ to determine the severity of their OCS. It consists of 2 subscales; obsession and compulsion. Total YBOCS score was used to assess the severity of OCS. In this study, clinically significant OCS was defined as total YBOCS score of 8 and above. YBOCS was suitable in the assessment of OCS in Schizophrenia ${ }^{34}$.

\section{Schizophrenia psychopathology}

Severity of schizophrenia symptoms were assessed using the Positive and Negative Syndrome Scale (PANSS) ${ }^{35}$ with the help of the Structure Clinical Interview version (SCI-PANSS). PANSS is a well-defined instrument to assess positive, negative as well as general symptoms in patients with schizophrenia. The higher the score, the more severe the psychopathologies. PANSS has 3 main sub-scales, namely positive, negative and general psychopathology. Overall, total PANSS score reflected the severity of schizophrenia.

\section{Depressive symptomatology}

In this study, we utilized the Calgary Depression Scale for Schizophrenia (CDSS) ${ }^{36}$ to assess participants' depressive symptoms. CDSS contains nine items. Each item consists of 4 options - 0 for absent, 1 for mild, 2 for moderate and 3 for severe symptoms. Higher score represents worse depressive symptoms.

\section{Suicidality assessment}

Suicidality in the present study was assessed using the Columbia Suicide Severity Rating Scale (screening version) (CSSR-S) ${ }^{37}$. Suicidality is defined as any suicidal behavior or ideation during the period of assessment according to the scoring and data analysis guide for CSSR-S. In this study, dichotomous outcome was used to ascertain presence or absence of suicidality.

\section{Quality of life assessment}

Participants' quality of life was assessed by using the 26-item World Health Organization Quality of Life - Brief Version (WHOQOLBREF). A validated Malay Version of WHOQOL-BREF38 was used in this study. Four domains were assessed, namely physical, psychological, social and environmental which denotes individuals' perception of their quality of life in each domain. Higher score denotes higher quality of life. WHOQOL-BREF (M) score was transformed into domain score comparable with the score used in WHOQOL-100 for data analysis according to the provided guideline.

\section{Statistical analysis}

All statistical analysis was performed using the Statistical Package for Social Sciences (SPSS) Version 22.0. Normal distribution of quantitative data was assessed using the Shapiro-Wilk test. Chi Square Test and Fisher Exact Test were used when necessary to compare categorical variables between schizophrenia with OCS and without OCS. Mann Whitney's U test was used to compare non-normally distributed continuous variables. Analysis of Covariance (ANCOVA) was performed to control the effect of chronicity of illness which was assessed empirically using duration of illness and the use of clozapine mainly for treatment resistance schizophrenia. Spearman's correlation was performed to examine the possible correlation between obsessive compulsive symptoms and schizophrenia psychopathology. Finally, logistic regression was carried out to analyze independent variables which were associated with OCS in schizophrenia. Level of significance was set at $\mathrm{p}<0.05$.

\section{Results}

\section{Demographic data}

A total of 220 outpatients were recruited. There was equal gender distribution between males (48.6\%) and females (51.4\%). Mean age 
for the participants was 43.7 years old $(\mathrm{SD}=12.4)$. Majority of the participants were Chinese (57.7\%), followed by Indian (23.6\%), Malay (15.6\%) and others (2.7\%). More than two third of the study participants were single $(70.0 \%)$ and their majority were unemployed (76.8\%). Patients were divided into two groups; schizophrenia with OCS and without OCS. There was no significant difference in terms of age, gender, race, education level, employment status, duration of illness and family history of any psychiatric illness between the two groups. In addition, the use of antipsychotic drug classes (both first and second generations) were of no significant difference between the schizophrenia patients with OCS and those without. However, significantly higher number of schizophrenia patients with OCS were taking Clozapine at the time of recruitment (odds ratio $=2.267$, $p=0.023)$ (Table 1).

Table 1. Comparison between Schizophrenia with and without OCS

\begin{tabular}{|c|c|c|c|c|c|}
\hline & & & & & \\
\hline & $\begin{array}{c}\text { Yes }(n=48) \\
N(\%)\end{array}$ & $\begin{array}{c}\text { No }(n=172) \\
N(\%)\end{array}$ & OR or U & $95 \% \mathrm{Cl}$ or $\mathrm{Z}$ & $P$ value \\
\hline Age, median & $38(98.14)^{c}$ & $44(113.95)^{c}$ & $3535.50 \mathrm{a}$ & $-1.523^{b}$ & 0.128 \\
\hline Male & $24(50.0)$ & $83(48.3)$ & 0.933 & $0.492-1.769$ & 0.871 \\
\hline Female & $24(50.0)$ & $89(51.7)$ & & & \\
\hline Chinese & $24(50.0)$ & $103(59.9)$ & 0.670 & $0.352-1.274$ & 0.249 \\
\hline Non-Chinese & $24(50.0)$ & $69(40.1)$ & & & \\
\hline Malay & $8(17.0)$ & $27(15.7)$ & 0.908 & $0.382-2.155$ & 0.824 \\
\hline Non-Malay & $39(83.0)$ & $145(84.3)$ & & & \\
\hline Indian & $15(31.3)$ & $37(21.5)$ & 1.658 & $0.815-3.375$ & 0.180 \\
\hline Non-Indian & $33(68.8)$ & $135(78.5)$ & & & \\
\hline Single & $35(72.9)$ & $119(69.2)$ & 0.834 & $0.408-1.703$ & 0.723 \\
\hline Married & $13(27.1)$ & $53(30.8)$ & & & \\
\hline Secondary and below education & $30(62.5)$ & $129(75.0)$ & 1.800 & $0.913-3.548$ & 0.102 \\
\hline Tertiary education & $18(37.5)$ & $43(25.0)$ & & & \\
\hline Employed & $10(20.8)$ & $41(23.8)$ & 0.841 & $0.385-1.834$ & 0.847 \\
\hline Unemployed & 38 (79.2) & $131(76.2)$ & & & \\
\hline Family history of mental illness & & & & & \\
\hline Yes & $20(41.7)$ & $55(32.0)$ & 1.519 & $0.788-2.932$ & 0.230 \\
\hline No & $28(58.3)$ & $117(68.0)$ & & & \\
\hline Duration of illness & & & & & \\
\hline Less than 5 years & $8(16.7)$ & $24(14.0)$ & 0.811 & $0.339-1.941$ & 0.646 \\
\hline 5 years and above & $40(83.3)$ & $148(86.0)$ & & & \\
\hline Antipsychotics & & & & & \\
\hline Typical & $6(12.5)$ & $42(24.4)$ & & & 0.171 \\
\hline Atypical & $35(72.9)$ & $112(65.1)$ & & & \\
\hline Combination & $7(14.6)$ & $18(10.5)$ & & & \\
\hline Clozapine & $30(62.5)$ & $36(20.9)$ & 2.267 & $1.137-4.520$ & $0.023^{*}$ \\
\hline Olanzapine & $8(16.7)$ & $30(17.4)$ & 0.947 & $0.403-2.226$ & 1.000 \\
\hline Risperidone & $9(18.8)$ & $50(29.1)$ & 0.563 & $0.254-1.248$ & 0.197 \\
\hline Antidepressant & $15(31.3)$ & $23(13.4)$ & 2.945 & $1.388-6.246$ & $0.008^{* *}$ \\
\hline Clinical variables & & & & & \\
\hline More than 5 admissions & $29(60.4)$ & $133(77.3)$ & 2.234 & $1.132-4.409$ & $0.026^{*}$ \\
\hline Antidepressant prescription & $15(31.3)$ & $23(13.4)$ & 2.945 & $1.388-6.246$ & $0.008^{* *}$ \\
\hline Suicidality & $27(56.3)$ & $45(26.2)$ & 3.629 & $1.868-7.048$ & $<0.001^{* *}$ \\
\hline PANSS Positive & $139.65^{c}$ & $102.37 \mathrm{c}$ & $2729.00 \mathrm{a}$ & $-3.603^{b}$ & $<0.001 * *$ \\
\hline PANSS Negative & $121.39_{c}$ & $107.46 \mathrm{c}$ & 3605.50a & $-1.343^{b}$ & 0.179 \\
\hline PANSS General & $133.22^{c}$ & $104.16^{c}$ & $3038.00^{a}$ & $-2.801^{b}$ & $0.005^{* *}$ \\
\hline PANSS Total & $133.22^{c}$ & $104.16 \mathrm{c}$ & $3037.50 \mathrm{a}$ & $-2.798^{b}$ & $0.005^{* *}$ \\
\hline CDSS & $134.31 \mathrm{c}$ & $103.85^{c}$ & $2985.00 \mathrm{a}$ & $-3.183^{b}$ & $0.001^{* *}$ \\
\hline Physical 0OL & $93.34 c$ & $115.29_{c}$ & 3304.50a & $-2.121 \mathrm{~b}$ & $0.034^{*}$ \\
\hline Psychological Q0L & $93.13 c$ & $115.35^{c}$ & $3294.00 \mathrm{a}$ & $-2.147 \mathrm{~b}$ & $0.032^{*}$ \\
\hline Social QOL & $105.22^{c}$ & $111.97 \mathrm{c}$ & $3874.50 \mathrm{a}$ & $-0.656^{b}$ & 0.512 \\
\hline Environmental QOL & $104.97 \mathrm{c}$ & $112.04 \mathrm{c}$ & $3862.50^{a}$ & $-0.683^{b}$ & 0.494 \\
\hline
\end{tabular}

a Median are compared with Mann Whitney's U test with b Z score.

b Median are compared with Mann Whitney's U test with b Z score.

c Non-normally distributed variables are expressed in mean rank.

Categorical variables with Fisher exact test.

* Significant level at $p<0.05$

** Significant level at $p<0.01$ 


\section{Prevalence of OCS in Schizophrenia}

In this study, we found that the prevalence of OCS in schizophrenia was $21.8 \%$ by using the total YBOCS score of 8 and above $(\mathrm{N}=48)$. The most common obsessions were aggression (8.2\%), contamination $(8.2 \%)$ and miscellaneous $(7.8 \%)$. For compulsion, the most commonly elicited compulsion was checking (8.2\%) and cleaning/ washing $(7.8 \%)$.

\section{Clinical variables of schizophrenia with OCS}

Spearman's correlation was performed and showed that obsession was significantly correlated with positive symptoms $(r=0.292$, $\mathrm{p}<0.001)$ and general symptoms $(\mathrm{r}=0.217, \mathrm{p}=0.001)$ of schizophrenia. For compulsions, results showed they were significantly correlated with positive symptoms $(\mathrm{r}=0.195, \mathrm{p}=0.004)$.

In this study, the group of schizophrenia patients with OCS documented significantly more hospitalizations $(\mathrm{OR}=2.234$, $\mathrm{p}=0.026$ ), higher number of prescription of antidepressants (odd ratio $=2.945, \mathrm{p}=0.008)$ and higher suicidality rate $(\mathrm{OR}=3.629, \mathrm{p}<$ $0.001)$. The same group of patients also scored higher in the PANSS positive subscale $(\mathrm{n}=48, \mathrm{U}=2729.00, \mathrm{p}<0.001)$, general subscale $(\mathrm{N}=48, \mathrm{U}=3038.00, \mathrm{p}=0.005)$, total PANSS $(\mathrm{N}=48, \mathrm{U}=3037.50$, $\mathrm{p}=0.005)$, and CDSS total score $(\mathrm{N}=48, \mathrm{U}=2985.00, \mathrm{p}=0.001)$. In addition, schizophrenia patients with OCS were also found to be associated with poorer physical quality of life $(\mathrm{N}=48, \mathrm{U}=3304.50$, $\mathrm{p}=0.034)$ and poorer psychological quality of life $(\mathrm{N}=48, \mathrm{U}=$ 3294.00, $\mathrm{p}=0.032$ ) (Table 1).

We continued to observe statistically significant differences between schizophrenia patients with OCS and those without, as reflected in the PANSS positive subscale, PANSS general symptoms subscale, PANSS total score and CDSS score (Table 2) after all the confounders were adjusted. For categorical clinical variables, logistic regression was performed by using illness chronicity as the covariate. Results showed the number of hospitalization; presence of suicidality and the use of antidepressant were significantly higher in schizophrenia patients with OCS (Table 3).

Table 2. Comparison between Schizophrenia with or without OCS in clinical variables (PANSS, CDSS and WHOQOL-BREF) using analysis of covariance (ANCOVA), Generalized Linear Models after controlled for chronicity of illness in the study sample

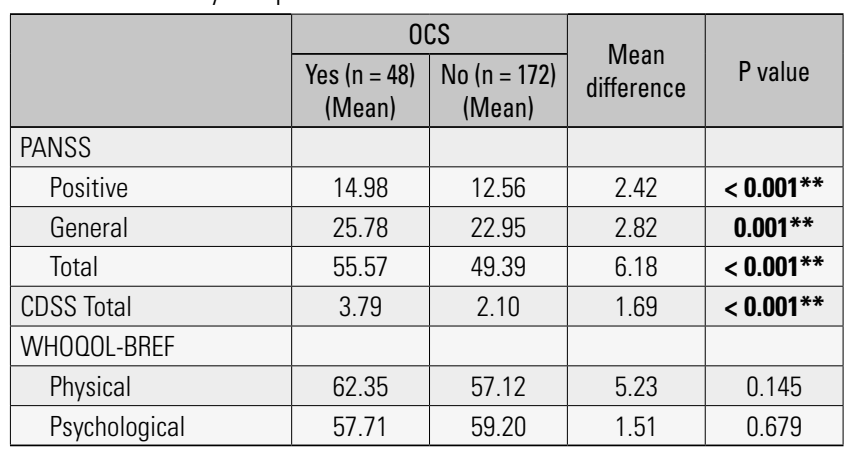

$P$ value significant level at $P<0.01 * *$

Table 3. Logistic regression for clinical variables using chronicity of illness as covariate: hospitalization, suicidality and use of antidepressant

\begin{tabular}{|l|c|c|c|c|}
\hline Variables & B & SE & Exp (B) & P value \\
\hline Hospitalization & 0.744 & 0.369 & 2.105 & $\mathbf{0 . 0 4 4}^{*}$ \\
\hline Suicidality & 1.257 & 0.348 & 3.516 & $<\mathbf{0 . 0 0 1}^{* *}$ \\
\hline Use of antidepressant & 0.974 & 0.392 & 2.650 & $\mathbf{0 . 0 1 3}^{*}$ \\
\hline
\end{tabular}

$P$ value significant level at $p<0.05^{*}, p<0.01^{* *}$.

\section{Discussion}

From this study, we found that the prevalence of OCS among schizophrenia patients in our setting is $21.8 \%$. This figure is very closed to the finding of a study done in another Asian country, Korea, which revealed a prevalence of $21.1 \%^{21}$. Nevertheless, our figure is lower when compared to the prevalence of $30.7 \%$ documented in a meta-analysis done in $2014^{2}$. Despite many studies conducted on this topic over the decades, there are still no universally accepted criteria for the diagnosis of OCS in schizophrenia. We believe that this shortcoming may have further complicated the estimation of prevalence of OCS in schizophrenia. In addition, studies which recruited inpatients $6,7,39$ and outpatients ${ }^{29,40,41}$ also showed a large difference in the prevalence of the comorbidity.

In terms of the socio-demographic factors between schizophrenia patients with OCS and those without, we observed that the duration of illness is not significantly associated with the presence of OCS in schizophrenia. This is in contrast with previous studies which reported that the longer the illness, the higher the prevalence of OCS in schizophrenia ${ }^{2,8,42}$. As expected, the usage of clozapine in schizophrenia was associated with high OCS, a finding consistent with previous studies $25,43-45$. It was postulated that high propensity of anti-serotonergic properties in clozapine could lead to or exacerbate serotonin deficiency, which subsequently trigger the onset of OCS and yield a full threshold OCD 22,24 . This iatrogenic mental disorder would pose additional challenge to the treatment. Clozapine is the only second generation antipsychotic that is effective in the treatment of resistant schizophrenia (TRS) ${ }^{46}$. Previous studies have identified few treatment options for clozapine induced OCS. One of the treatments is to combine Aripiprazole with Clozapine. Aripiprazole is a second-generation antipsychotic with few trials already carried out showing its effectiveness in treating Clozapine induced OCS in TRS. TRS patients who received the combination of AripiprazoleClozapine not only had reduced total YBOCS score at one month after treatment, but also showed better tolerability ${ }^{47}$ and improvement in quality of life ${ }^{48}$ as compared to Clozapine-placebo group ${ }^{49}$. In fact, by adding on a Selective Serotonin Reuptake Inhibitor (SSRI) it is more effective in treating induced OCS, in cases of treatmentresistant schizophrenia which normally only responds to clozapine. Escitalopram $^{50}$, fluvoxamine ${ }^{51}$ and fluoxetine ${ }^{52}$ had shown their effectiveness in treating induced OCS. However, more trials needed to be done to identify the most effective medication to be used in clozapine induced OCS in treating TRS.

In some of the previous studies, it was reported that schizophrenia patients with OCS were associated with more severe psychopathology 7,8 and depressive symptoms $9,10,40,53$. Our study findings are consistent with these previous studies, as our patients also had more psychotic symptoms and higher depressive symptom scores. These findings were not influenced by chronicity of illness in the participants, as well as the concurrent use of Clozapine. Furthermore, the presence of OCS had been associated with higher suicidality in patients with schizophrenia ${ }^{12,54}$ in which our study also revealed similar finding. This is clinically important as practicing clinicians ought to be aware of the impact of OCS, hence be more vigilant when assessing this group of patients for suicidality.

Our study also noted poorer physical and psychological quality of life among these schizophrenia patients with OCS. These findings are in line with a recent study conducted in Amsterdam which revealed that comorbid OCS in schizophrenia was associated with a lower mean across all domains, namely subjective wellbeing, social integration, emotional regulation, physical and mental health ${ }^{41}$. Similar findings were also reported by Tiryaki and Özkorumak in their study involving 62 patients with schizophrenia and OCS ${ }^{6}$. Thus, we need to create awareness among all practicing clinicians when they treat comorbid OCS, as this will contribute in the improvement of patients' quality of life 55 .

Some limitations were present in our study. Firstly, by the nature of a cross-sectional design of our study, we were unable to explore the causal relationship between the presence of OCS and clinical 
variables. Secondly, there was no universally accepted criteria to diagnose OCS in schizophrenia. Previous studies had used different criteria to diagnose OCS which produced variabilities in their study outcome. Thirdly, since this study was done at a local outpatient setting, the findings could not be generalized to other parts of the world. Thus, further systematic studies are needed in near future to explore the diagnostic criteria for OCS and a prospective study design to determine the causal relationship of OCS in schizophrenia.

Schizophrenia with OCS remains prevalent in many parts of the world. The awareness of this non-schizophrenia psychopathological comorbid among practicing clinicians is crucial as this is a different entity of psychiatric disorder needing special attention and treatment approach. Gross oversight of this comorbid will result in severe depressive symptoms and higher suicidality. In addition, clinicians need to also be aware of the pro-obsessive effect of Clozapine in the treatment of refractory schizophrenia. It is our hope that more attention will be given to this prevalent condition through rigorous research within this area in near future.

\section{Competing interests}

The author(s) declared that they have no competing interests.

\section{Acknowledgements}

We would like to thank the University of Malaya Research Management Centre for providing Postgraduate Research Fund (PO048-2014B) to fund this study.

\section{References}

1. Berman I, Merson A, Viegner B, Losonczy MF, Pappas D, Green AI. Obsessions and compulsions as a distinct cluster of symptoms in schizophrenia: a neuropsychological study. J Nerv Ment Dis. 1998;186(3):150-6.

2. Swets M, Dekker J, van Emmerik-van Oortmerssen K, Smid GE, Smit F, de Haan $\mathrm{L}$, et al. The obsessive compulsive spectrum in schizophrenia, a meta-analysis and meta-regression exploring prevalence rates. Schizophr Res. 2014;152(2-3):458-68.

3. Mulky P, Raghavan DV, Maheshwari S, Kannan P, Jeyaprakash R. Obsessive-Compulsive symptoms in patients with Schizophrenia attending tertiary care centre in South India. Stanley Medical J. 2016;2(4):2-7.

4. Poyurovsky M, Fuchs C, Weizman A. Obsessive-compulsive disorder in patients with first-episode schizophrenia. Am J Psychiatry. 1999;156(12):1998-2000.

5. Bottas A, Cooke RG, Richter MA. Comorbidity and pathophysiology of obsessive-compulsive disorder in schizophrenia: is there evidence for a schizo-obsessive subtype of schizophrenia? J Psychiatry Neurosci. 2005;30(3):187-93.

6. Tiryaki A, Özkorumak E. Do the obsessive-compulsive symptoms have an effect in schizophrenia? Compr Psychiatry. 2010;51(4):357-62.

7. Owashi T, Ota A, Otsubo T, Susa Y, Kamijima K. Obsessive-compulsive disorder and obsessive-compulsive symptoms in Japanese inpatients with chronic schizophrenia - a possible schizophrenic subtype. Psychiatry Res. 2010;179(3):241-6.

8. Kim SW, Jeong BO1, Kim JM, Shin IS, Hwang MY, Paul Amminger G, et al. Associations of obsessive-compulsive symptoms with clinical and neurocognitive features in schizophrenia according to stage of illness. Psychiatry Res. 2015;226(1):368-75.

9. Hagen K, Hansen B, Joa I, Larsen TK. Prevalence and clinical characteristics of patients with obsessive-compulsive disorder in first-episode psychosis. BMC Psychiatry. 2013;13:156.

10. Niendam TA, Berzak J, Cannon TD, Bearden CE. Obsessive compulsive symptoms in the psychosis prodrome: Correlates of clinical and functional outcome. Schizophr Res. 2009;108(1-3):170-5.

11. Rajkumar RP, Reddy YCJ, Kandavel T. Clinical profile of "schizo-obsessive" disorder: a comparative study. Compr Psychiatry. 2008;49(3):262-8.

12. Sevincok L, Akoglu A, Kokcu F. Suicidality in schizophrenic patients with and without obsessive-compulsive disorder. Schizophr Res. 2007;90(13):198-202.
13. Poyurovsky M, Zohar J, Glick I, Koran LM, Weizman R, Tandon R, et al. Obsessive-compulsive symptoms in schizophrenia: implications for future psychiatric classifications. Compr Psychiatry. 2012;53(5):480-3.

14. Gupta S, Andreasen NC, Arndt S, Flaum M, Schultz SK, Hubbard WC, et al. Neurological soft signs in neuroleptic-naive and neuroleptic-treated schizophrenic patients and in normal comparison subjects. Am J Psychiatry. 1995;152(2):191-6.

15. Mohr F, Hubmann W, Albus M, Franz U, Hecht S, Scherer J, et al. Neurological soft signs and neuropsychological performance in patients with first episode schizophrenia. Psychiatry Res. 2003;121(1):21-30.

16. Sevincok L, Akoglu A, Topaloglu B, Arslantas H. Neurological soft signs in schizophrenic patients with obsessive-compulsive disorder. Psychiatry Clin Neurosci. 2004;58(3):274-9.

17. Sevincok L, Akoglu A, Arslantas H. Schizo-obsessive and obsessive-compulsive disorder: Comparison of clinical characteristics and neurological soft signs. Psychiatry Res. 2006;145(2-3):241-8.

18. Faragian S, Pashinian A, Fuchs C, Poyurovsky M. Obsessive-compulsive symptom dimensions in schizophrenia patients with comorbid obsessive-compulsive disorder. Prog Neuropsychopharmacol Biol Psychiatry. 2009;33(6):1009-12.

19. Bloch MH, Landeros-Weisenberger A, Rosario MC, Pittenger C, Leckman JF. Meta-analysis of the symptom structure of obsessive-compulsive disorder. Am J Psychiatry. 2008;165(12):1532-42.

20. Lykouras L, Alevizos B, Michalopoulou P, Rabavilas A. Obsessive-compulsive symptoms induced by atypical antipsychotics. A review of the reported cases. Prog Neuropsychopharmacol Biol Psychiatry. 2003;27(3):333-46.

21. Lim M, Park DY, Kwon JS, Joo YH, Hong KS. Prevalence and clinical characteristics of obsessive-compulsive symptoms associated with atypical antipsychotics. J Clin Psychopharmacol. 2007;27(6):712-3.

22. Fonseka TM, Richter MA, Müller DJ. Second generation antipsychotic-induced obsessive-compulsive symptoms in schizophrenia: a review of the experimental literature. Curr Psychiatry Rep. 2014;16(11):510.

23. Schirmbeck F, Rausch F, Englisch S, et al. Differential effects of antipsychotic agents on obsessive - Compulsive symptoms in schizophrenia: A longitudinal study. J Psychopharmacol. 2013;27(4):349-57.

24. Baker RW, Chengappa KNR, Baird JW, Steingard S, Christ MAG, Schooler NR. Emergence of obsessive compulsive symptoms during treatment with clozapine. J Clin Psychiatry. 1992;53(12):439-42.

25. Doyle M, Chorcorain AN, Griffith E, Trimble T, O'Callaghan E. Obsessive compulsive symptoms in patients with Schizophrenia on Clozapine and with Obsessive Compulsive disorder: a comparison study. Compr Psychiatry. 2014;55(1):130-6.

26. Pampaloni I, Tyagi H, Drummond L. Augmentation in profound, SRI-refractory OCD: Is aripiprazole superior to other to other dopamine blockers? Eur Psychiatry. 2017;41:S322.

27. Masi G, Pfanner C, Brovedani P. Antipsychotic augmentation of selective serotonin reuptake inhibitors in resistant tic-related obsessive-compulsive disorder in children and adolescents: A naturalistic comparative study. J Psychiatr Res. 2013;47(8):1007-12.

28. Üçok A, Ceylan ME, Tihan AK, Lapçin S, Ger C, Tükel R. Obsessive compulsive disorder and symptoms may have different effects on schizophrenia. Prog Neuropsychopharmacol Biol Psychiatry. 2011;35(2): 429-33.

29. Frías A, Palma C, Farriols N, Becerra C, Álvarez A, Cañete J. Neuropsychological profile and treatment-related features among patients with comorbidity between schizophrenia spectrum disorder and obsessivecompulsive disorder: Is there evidence for a "schizo-obsessive" subtype? Psychiatry Res. 2014;220(3):846-54.

30. Poyurovsky M, Hramenkov S, Isakov V, Rauchverger B, Modai I, Schneidman M, et al. Obsessive-compulsive disorder in hospitalized patients with chronic schizophrenia. Psychiatry Res. 2001;102(1):49-57.

31. Bottas A, Cooke RG, Richter MA. Comorbidity and pathophysiology of obsessive-compulsive disorder in schizophrenia: is there evidence for a schizo-obsessive subtype of schizophrenia? J Psychiatry Neurosci. 2005;30(3):187-93.

32. Devi S, Rao NP, Badamath S, Chandrashekhar CR, Janardhan Reddy YC. Prevalence and clinical correlates of obsessive-compulsive disorder in schizophrenia. Compr Psychiatry. 2015;56:141-8.

33. Goodman WK, Price LH, Rasmussen SA, Mazure C, Fleischmann RL, Hill CL, et al. The Yale-Brown Obsessive Compulsive Scale. I. Development, use, and reliability. Arch Gen Psychiatry. 1989;46(11):1006-11. 
34. de Haan L, Hoogeboom B, Beuk N, Wouters L, Dingemans PM, Linszen DH. Reliability and validity of the Yale-Brown Obsessive-Compulsive Scale in schizophrenia patients. Psychopharmacol Bull. 2006;39(1):25-30.

35. Kay SR, Opler LA, Lindenmayer JP. Reliability and validity of the positive and negative syndrome scale for schizophrenics. Psychiatry Res. 1988;23(1):99-110.

36. Addington D, Addington J, Atkinson M. A psychometric comparison of the Calgary Depression Scale for Schizophrenia and the Hamilton Depression Rating Scale. Schizophr Res. 1996;19(2-3):205-12.

37. Posner K, Brown GK, Stanley B, Brent DA, Yershova KV, Oquendo MA, et al. The Columbia-Suicide Severity Rating Scale: initial validity and internal consistency findings from three multisite studies with adolescents and adults. Am J Psychiatry. 2011;168(12):1266-77.

38. Hasanah CI, Naing L, Rahman AR. World Health Organization Quality of Life Assessment: brief version in Bahasa Malaysia. Med J Malaysia. 2003;58(1):79-88.

39. Poyurovsky M, Bergman J, Weizman R. Obsessive-compulsive disorder in elderly schizophrenia patients. J Psychiatr Res. 2006;40(3):189-91.

40. Ongur D, Goff DC. Obsessive-compulsive symptoms in schizophrenia: associated clinical features, cognitive function and medication status. Schizophr Res. 2005; 75(2-3):349-62.

41. de Haan L, Sterk B, van der Valk R. Presence of obsessive compulsive symptoms in first-episode schizophrenia or related disorders is associated with subjective well-being and quality of life. Early Interv Psychiatry. 2013;7(3):285-90.

42. Tibbo P, Kroetsch M, Chue P, Warneke L. Obsessive-compulsive disorder in schizophrenia. J Psychiatr Res. 2000;34(2):139-46.

43. Goh KK, Chiu Y-H, Shen WW, Yeh E-K. Dilemma in Treating Clozapine-induced Obsessive-compulsive Symptoms in a Patient with Schizophrenia. J Exp Clin Med. 2013;3(5):118-19.

44. Scheltema BA, Swets M, Machielsen M, Korver N. Obsessive-compulsive symptoms in patients with schizophrenia: a naturalistic cross-sectional study comparing treatment with clozapine, olanzapine, risperidone, and no antipsychotics in 543 patients. J Clin Psychiatry. 2012;73(11):1395-402.

45. Galvez-Buccollini JA, Fiestas F, Herrera P, Vega-Dienstmaier JM, Guimas B, Mazzotti G. [Obsessive-compulsive symptoms in schizophrenia during treatment with clozapine and conventional antipsychotic drugs]. Actas Esp Psiquiatr. 2004;32(4):211-5.

46. Kane J, Honigfeld G, Singer J, Meltzer H. Clozapine for the treatment-resistant schizophrenic: A double-blind comparison with chlorpromazine. Arch Gen Psychiatry. 1988;45(9):789-96.

47. Eryılmaz G, Hızlı Sayar G, Ozten E, Gögcegöz Gül I, Karamustafalığlu O. Aripirazole augmentation in clozapine-associated obsessive-compulsive symptoms in schizophrenia. Ann Gen Psychiatry. 2013;12(1):40.

48. Glick ID, Poyurovsky M, Ivanova O, Koran LM. Aripiprazole in schizophrenia patients with comorbid obsessive-compulsive symptoms: an open-label study of 15 patients. J Clin Psychiatry. 2008;69(12):1856-9.

49. Chang JS, Ahn YM, Park HJ, Lee KY, Kim SH, Kang UG, et al. Aripiprazole augmentation in clozapine-treated patients with refractory schizophrenia: an 8-week, randomized, double-blind, placebo-controlled trial. J Clin Psychiatry. 2008;69(5):720-31.

50. Stryjer R, Dambinsky Y, Timinsky I, Green T, Kotler M, Weizman A, et al. Escitalopram in the treatment of patients with schizophrenia and obsessive-compulsive disorder: An open-label, prospective study. Int Clin Psychopharmacol. 2013 Mar;28(2):96-8.

51. Poyurovsky M, Hermesh H, Weizman A. Fluvoxamine treatment in clozapine-induced obsessive-compulsive symptoms in schizophrenic patients. Clin Neuropharmacol. 1996;19(4):305-13.

52. Levy E, Margolese HC, Sultan S, Chouinard G. Obsessive-Compulsive Symptoms in Schizophrenia Induced by Risperidone and Responding to Fluoxetine. Can J Psychiatry. 2003;48(10):709-10.

53. DeVylder JE, Oh AJ, Ben-David S, Azimov N, Harkavy-Friedman JM Corcoran CM. Obsessive compulsive symptoms in individuals at clinical risk for psychosis: association with depressive symptoms and suicidal ideation. Schizophr Res. 2012;140(1-3):110-3.

54. Ucok A, Tukel R, Ozgen G, Saylan M, Uzuner G. [Frequency of obsessive compulsive symptoms and disorder in patients with schizophrenia: importance for prognosis]. Encephale. 2006;32(1 Pt 1):41-4.

55. Ucok A, Kivrak Tihan A, Karadayi G, Tukel R. Obsessive compulsive symptoms are related to lower quality of life in patients with Schizophrenia. Int J Psychiatry Clin Pract. 2014;18(4):243-7. 